\title{
Lognormal Distribution of Cellular Uptake of Radioactivity: Statistical Analysis of $\alpha$-Particle Track Autoradiography
}

\author{
Prasad V.S.V. Neti and Roger W. Howell \\ Division of Radiation Research, Department of Radiology, New Jersey Medical School, University of Medicine and Dentistry of \\ New Jersey, Newark, New Jersey
}

Recently, the distribution of radioactivity among a population of cells labeled with ${ }^{210} \mathrm{Po}$ was shown to be well described by a lognormal (LN) distribution function (J Nucl Med. 2006;47:10491058 ) with the aid of autoradiography. To ascertain the influence of Poisson statistics on the interpretation of the autoradiographic data, the present work reports on a detailed statistical analysis of these earlier data. Methods: The measured distributions of $\alpha$-particle tracks per cell were subjected to statistical tests with Poisson, LN, and Poisson-lognormal (P-LN) models. Results: The LN distribution function best describes the distribution of radioactivity among cell populations exposed to 0.52 and $3.8 \mathrm{kBq} / \mathrm{mL}$ of ${ }^{210} \mathrm{Po}$-citrate. When cells were exposed to $67 \mathrm{kBq} / \mathrm{mL}$, the P-LN distribution function gave a better fit; however, the underlying activity distribution remained lognormal. Conclusion: The present analysis generally provides further support for the use of LN distributions to describe the cellular uptake of radioactivity. Care should be exercised when analyzing autoradiographic data on activity distributions to ensure that Poisson processes do not distort the underlying LN distribution.

Key Words: Poisson; log normal; Poisson-log normal; ${ }^{210} \mathrm{Po}$; autoradiography; $\alpha$-particle tracks; reduced chi-square

J Nucl Med 2008; 49:1009-1016

DOI: 10.2967/jnumed.107.048843

\section{A} utoradiography has been used for decades to quantify the distribution of radioactivity in tissues after administration of radiochemicals $(1-6)$. Recently, $\alpha$-particle track autoradiography was used to determine the distribution of ${ }^{210} \mathrm{Po}-$ citrate among a clonal cell population of Chinese hamster V79 lung fibroblasts (7). Analysis of the data revealed a lognormal (LN) distribution of cellular activity, a distribution that can have a substantial effect on the biologic response of

Received Nov. 5, 2007; revision accepted Dec. 6, 2007.

For correspondence or reprints contact: Roger W. Howell, Department of Radiology, MSB F-451, New Jersey Medical School, University of Medicine and Dentistry of New Jersey, 185 S. Orange Ave., Newark, NJ 07103.

E-mail: rhowell@umdnj.edu

COPYRIGHT @ 2008 by the Society of Nuclear Medicine, Inc. the cell population $(7,8)$. To arrive at this distribution, the number of $\alpha$-particle tracks per cell was scored in more than 1,000 cells. However, it was necessary to limit the number of counted tracks per cell to fewer than 10 to ensure accurate scoring. The statistical uncertainties associated with measuring these low numbers of tracks per cell can affect the measuredtrack distribution and thereby potentially distort the underlying activity distribution among the cell population $(9,10)$.

As pointed out by Kvinnsland et al. (9), it is the statistical nature of radioactive decay that can influence the measured distribution of $\alpha$-particle tracks, particularly at the low numbers of tracks per cell studied by Neti et al. (7). Therefore, it may be necessary to "peel off" the distribution associated with the statistics of radioactive decay to arrive at the underlying activity distribution. It has been hypothesized that in radioactive decay, all atoms are identical and independent, and the chance for an atom to disintegrate during a given time interval is the same for all time intervals of equal size. Accordingly, after the discovery of radioactivity, initial experiments suggested that the decay process, and particle counting associated with its observation, is well approximated by simple Poisson processes. Subsequently, during a study of $\alpha$-particle emission rates, a quantum self-interference phenomenon was proposed to account for $1 / f$ fluctuations (11). Experiments showed that $\alpha$-emission is in fact not a simple Poisson process, and particle counting is not adequately described by Poisson statistics (12). However, another report states that $1 / f$ fluctuations are not present in the decay of ${ }^{210} \mathrm{Po}$ at decay frequencies in excess of $10^{-6} \mathrm{~Hz}$ (13). The mean track numbers for the range of ${ }^{210} \mathrm{Po}$-citrate concentrations discussed in the present report fall in the window of approximately $0.16-1.6 \times 10^{-6} \mathrm{~Hz}$. Consequently, it is unlikely that $1 / f$ fluctuations play a significant role in interpreting our experimental $\alpha$-particle track data (7). Nevertheless, Poisson statistics may play a role; it is necessary, therefore, to examine whether the LN distribution obtained from our ${ }^{210} \mathrm{Po} \alpha$-particle track data is influenced by the Poisson distribution associated with its decay. Accordingly, in this report we subject our $\alpha$-particle track data to statistical tests with Poisson, LN, and Poisson-lognormal (P-LN) distribution functions (14). 


\section{MATERIALS AND METHODS}

Summary of Published Experimental Data and Analyses

The methods used to obtain the $\alpha$-particle track data statistically analyzed in the current study are explained in detail in an earlier report (7). Briefly, cultured cells were labeled with ${ }^{210} \mathrm{Po}-$ citrate, washed, and coated with autoradiographic emulsion. Decays were allowed to accumulate for various times to obtain scoreable track data that covered the entire range of cellular activities encountered in a given labeled cell population. Accordingly, the emulsion was developed for 2 or 3 different exposure times. For each emulsion exposure time, 500-1,200 cells were examined, and the number of tracks in each cell was recorded. Cells with 0-9 tracks per cell were scored as such. Cells with more than 9 tracks could not be accurately counted and were simply scored as greater than 9 tracks. The entire process was repeated for ${ }^{210} \mathrm{Po}$-citrate-labeling concentrations of $0.52,3.8$, and $67 \mathrm{kBq} / \mathrm{mL}$, which resulted in mean cellular activities of $0.054,0.12$, and 1.8 $\mathrm{mBq}$ per cell. The numbers of tracks per cell from the short exposure times were decay-corrected to the longest exposure time and then normalized. All datasets for a given labeling concentration were then combined into a single convolved track distribution with the longest exposure time data providing the distribution for the lower multiplicity of convolved tracks and the short exposure time data providing the distribution of the higher multiplicity. A nonlinear least-squares (LS) fit of the resulting convolved track distribution was performed with an $\mathrm{LN}$ function (7).

\section{Experimental Mean Number of $\alpha$-Particle Tracks per Cell}

The arithmetic mean number of tracks per cell $\langle n\rangle$ can be calculated for the track data corresponding to the shortest emulsion exposure times for each labeling concentration. These data have no cells with more than 9 tracks, and therefore the mean is obtained by simply tallying the total number of tracks and dividing by the number of cells. A different approach was required to calculate $\langle n\rangle$ for the datasets corresponding to longer emulsion exposure times that contained cells with more than 9 tracks. Regardless of the mathematic nature of the track distribution, the total number of tracks $n_{\text {total }}$ recorded in the cell population at time point $t_{1}$ can be projected to a later time point $t_{2}$ according to Equation 1,

$$
n_{\text {total }}\left(t_{1} \Rightarrow t_{2}\right)=n_{\text {total }}\left(t_{1}\right) \frac{\left(1-e^{-0.693 t_{2} / T_{\mathrm{p}}}\right)}{\left(1-e^{-0.693 t_{1} / T_{\mathrm{p}}}\right)}
$$

where $T_{\mathrm{p}}$ is the physical half-life of the radionuclide $\left(T_{\mathrm{p}}=138 \mathrm{~d}\right.$ for ${ }^{210} \mathrm{Po}$ ). In the same manner, $\left\langle n\left(t_{1}\right)>\right.$ (shortest exposure time with all cells having $<9$ tracks) can be used to calculate $\left\langle n\left(t_{2}\right)\right\rangle$ (longer exposure time with some cells having $>9$ tracks).

\section{Statistical Distributions and Analyses}

Poisson Distribution. If each cell in the population had the same activity, one would anticipate a Poisson distribution of tracks per cell. A $1 / f$ distribution may be expected according to one study (12); however, another report indicates that $1 / f$ fluctuations are not present in the decay of ${ }^{210} \mathrm{Po}$ for frequencies in excess of $10^{-6} \mathrm{~Hz}$ (13), a frequency range that encompasses our data. Thus, in this report, the Poisson distribution is assumed to adequately describe the statistical distribution associated with the decay of ${ }^{210} \mathrm{Po}$. After finding $\langle n\rangle$ for each set of track distribution data, we calculated the Poisson probability of $n$ discrete tracks per cell, given by

$$
\mathrm{P}_{P}(n)=e^{-<n>} \frac{<n>^{n}}{n !}(n=0,1,2, \ldots) . \quad \text { Eq. } 2
$$

For noninteger values of $n$, which may result because of decay correction (Eq. 1), truncated values are used to calculate the probability.

$L N$ Distribution. The LN probability density function is given by

$$
\mathrm{P}_{L N}(n)=\frac{g}{n \sigma} e^{\frac{-\left(\ln n-\mu_{n}\right)^{2}}{2 \sigma^{2}}}, n>0, \quad \text { Eq. } 3
$$

where $\mu_{n}$ is the scale parameter, $\sigma$ is the shape parameter, and $g$ is a constant. The scale parameter is related to the mean according to $\mu_{n}=\ln \langle n\rangle-\sigma^{2} / 2$. The properties of this function and its use in analysis of the distribution of tracks per cell are given in a previous study (7). Detailed information on LN distributions can be found in other studies $(15,16)$ as well.

$P$-LN Distribution. The P-LN compound probability of obtaining a realization $n$ and all its possible Poisson realizations $k$ is given in an earlier study (17),

$$
\mathrm{P}_{P-L N}(n \mid<n>)=\sum_{k=1}^{\infty} \frac{1}{\sqrt{2 \pi} \sigma n} e^{-\frac{\left(\ln \frac{n}{k}-\frac{\sigma^{2}}{2}\right)^{2}}{2 \sigma^{2}}} e^{-<n>} \frac{<n>^{n}}{n !} .
$$

$\hat{\chi}^{2}$ Analyses. To quantitatively assess which of the above statistical distributions best describes the observed track distribution data, a reduced $\chi^{2}$ analysis $\left(\hat{\chi}^{2}\right)$ was performed. This quantity is given by Equation 5,

$$
\hat{\chi}^{2}=\frac{1}{v} \sum_{i=1}^{i_{\max }} \frac{\left(n_{i}-n_{\text {expected }, i}\right)^{2}}{n_{i}}
$$

where $n_{i}$ is the number of tracks per cell for the $i$ th experimental data, $n_{\text {expected }, i}$ is the expected value based on the assumed distribution, $\nu$ is the degree of freedom $\left(\nu=i_{\max }-\mathrm{p}-1\right)$, and $\mathrm{p}$ is the number of parameters in the assumed distribution.

LS Fit. LS fits of the data to an LN distribution function (Eq. 3) were performed with software (Sigmaplot; Systat Software). The LS fit of the LN distribution function to the data corresponding to emulsion exposure times where more than 9 tracks were observed was performed by 2 methods. In the first method, $\langle n\rangle$ was calculated by decay-correcting $\left\langle n\left(t_{1}\right)\right\rangle$ to obtain $\left\langle n\left(t_{2}\right)\right\rangle$ and then constraining $\left\langle n\left(t_{2}\right)\right\rangle$ to obtain $\sigma$. In the second method, $\langle n\rangle$ and $\sigma$ were allowed to vary. No constraints were placed on the LS fit of the LN distribution function to the convolved data.

\section{RESULTS}

\section{Statistical Analysis for Cells Exposed to $0.52 \mathrm{kBq} / \mathrm{mL}$}

To study the effect of Poisson statistics on our analyses, the 3 discrete raw datasets from Figure $3 \mathrm{~A}$ in an earlier study (7) $(0.52 \mathrm{kBq} / \mathrm{mL})$ are first revisited. The 3 sets of track distributions were acquired from autoradiographs that were developed at 7, 26, and $52 \mathrm{~d}$, as shown in Figures 1A- 


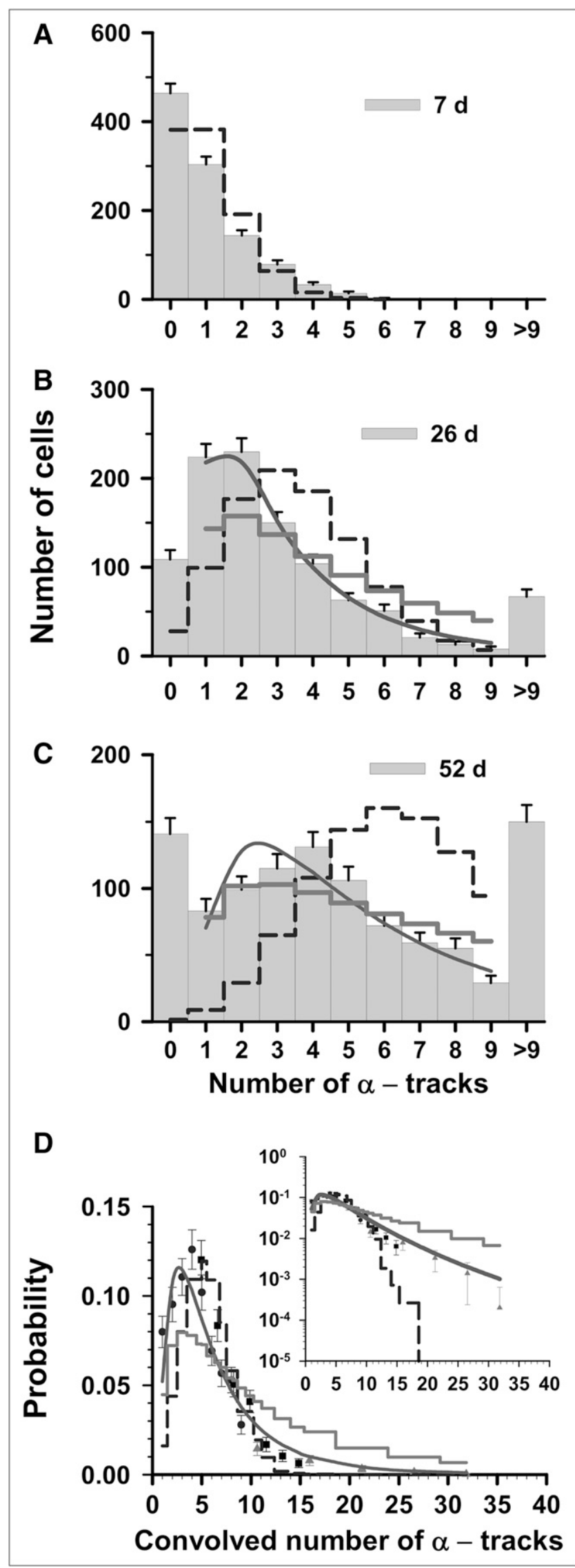

$1 \mathrm{C}$ of the current study. Each set of track distribution data includes the number of cells scored with 0-9 tracks per cell as well as the number of cells with an unscoreable number of tracks ( $>9$ tracks). The $t=7 \mathrm{~d}$ data have a maximum of 6 tracks in any given cell, and no cells have more than 9 tracks. The mean value for the $t=7 \mathrm{~d}$ data is 1.0 tracks per cell. The values of $\langle n\rangle$ for $t=26 \mathrm{~d}$ and $t=52 \mathrm{~d}$ cannot be obtained directly from the respective datasets because some of the cells have greater than 9 tracks. Thus, the projected $\langle n\rangle$ values obtained with Eq. 1 are 3.6 for the 26-d data and 6.7 for the 52-d data. Using these $<n>$ values, the Poisson probabilities for each of 0-9 discrete tracks per cell are calculated at $t=7,26$, and $52 \mathrm{~d}$. These probabilities can be used to assess the extent to which our experimental data represent a Poisson distribution. As shown in Figure 1A, the $t=7 \mathrm{~d}$ experimental data follow the general trend of a Poisson distribution, which suggests that Poisson statistics may have some impact on these data. The $t=26 \mathrm{~d}$ (Fig. 1B) and $t=52 \mathrm{~d}$ (Fig. 1C) datasets do not follow a Poisson distribution, although this does not rule out a Poisson component in these distributions.

To assess the possible Poisson component, we return to the $t=52 \mathrm{~d}$ dataset. This is the primary dataset, covers the range of 0-9 tracks in our convolved data, and primarily dictates the shape of the probability distribution. For the $t=52 \mathrm{~d}$ data, the $\hat{\chi}^{2}$ values for the Poisson, LN, and P-LN distributions were $149,3.2$, and 6.5. The latter 2 values were obtained via a $\hat{\chi}^{2}$ minimization procedure with the $\sigma$. The values of $\sigma$ were 0.81 and 1.1 for the LN and P-LN distributions, respectively. The lowest $\hat{\chi}^{2}$ value was obtained for the LN distribution as shown in Table 1, suggesting that Poisson corrections are not required for the $t=52 \mathrm{~d}$ data. Furthermore, the $\sigma$ obtained with the LN distribution corresponding to the discrete $t=52 \mathrm{~d}$ data was the same as that obtained by LS fit of the convolved data, which included the $t=7,26$, and $52 \mathrm{~d}$ data.

Now that the LN distribution has been firmly established with the primary $t=52 \mathrm{~d}$ data, we return to the ancillary $t=7$ and $26 \mathrm{~d}$ data where the Poisson distribution may play a more significant role. These data represent cells from the tail of the LN distribution and therefore contain little

FIGURE 1. Statistical analysis of $\alpha$-particle track distribution in V79 lung fibroblasts that were labeled in culture medium containing $0.52 \mathrm{kBq}$ of ${ }^{210} \mathrm{Po}$-citrate per milliliter. (A-C) Vertical bars with SEs represent experimental track distributions (discrete) when scored after decays had been allowed to accumulate for 7, 26, and $52 \mathrm{~d}(7)$. (D) Data points represent normalized convolution of experimental track data obtained at $7 \mathrm{~d}(\boldsymbol{\Delta}), 26 \mathrm{~d}$ $(\varpi)$, and $52 \mathrm{~d}(\bullet)$. Error bars represent SEs. In each panel, predicted probabilities based on Poisson, P-LN, and LN functions are represented by dashed step line, thick solid step line, and solid curve, respectively. Parameters of 3 probability density functions are enumerated in Table 1. Inset plots ordinates on log scale to compare trends of these probability functions relative to experimental data at high numbers of convolved tracks. 
TABLE 1

Statistical Analysis of ${ }^{210} \mathrm{Po} \alpha$-Particle Track Distribution Data (7) Using Poisson, LN, and P-LN Functions

\begin{tabular}{|c|c|c|c|c|c|c|c|}
\hline $\begin{array}{c}\text { Labeling } \\
\text { concentration } \\
(\mathrm{kBq} / \mathrm{mL})^{\star}\end{array}$ & Exposure time (d) & Function & $\begin{array}{c}<n>t \\
\text { (tracks/cell) }\end{array}$ & $\sigma^{\ddagger}$ & $v$ & $\hat{\chi}^{2}$ & $R^{2}$ \\
\hline \multirow[t]{9}{*}{$0.52 \pm 0.051$} & $52 \mathrm{~d}$ & Poisson ) & $6.7 \S$ & - & 7 & 149 & \\
\hline & & LN & & 0.81 & 6 & 3.2 & - \\
\hline & & P-LN J & & 1.1 & 6 & 6.5 & \\
\hline & & Constrained, LS fit to LN & $6.7^{\S \pm 1.1}$ & $0.80 \pm 0.097$ & 5 & 3.9 & 0.78 \\
\hline & & LS fit to LN & $8.2 \pm 1.7$ & $0.88 \pm 0.11$ & 6 & 3.2 & 0.81 \\
\hline & Convolved $(52,26,7$ d) & Poisson ) & 5.5 & - & 19 & $7.7 \mathrm{E}+07$ & \\
\hline & & LN & & 0.71 & 18 & 3.6 & - \\
\hline & & P-LN & & 0.96 & 18 & 12 & \\
\hline & & LS fit to LN & $7.1 \pm 0.63$ & $0.80 \pm 0.056$ & 18 & 3.1 & 0.92 \\
\hline \multirow[t]{9}{*}{$3.8 \pm 0.36$} & $4 d$ & Poisson & $7.3^{\S}$ & - & 7 & 25 & \\
\hline & & LN $\quad\}$ & & 0.64 & 6 & 0.5 & - \\
\hline & & P-LN $\quad$ S & & 0.60 & 6 & 1.1 & \\
\hline & & Constrained, LS fit to LN & $7.3^{\S} \pm 0.32$ & $0.61 \pm 0.029$ & 5 & 1.2 & 0.98 \\
\hline & & LS fit to $L N$ & $7.4 \pm 0.33$ & $0.62 \pm 0.029$ & 6 & 0.9 & 0.98 \\
\hline & Convolved $(0.67,4 \mathrm{~d})$ & Poisson & 6.1 & - & 16 & $1.2 \mathrm{E}+07$ & \\
\hline & & LN & & 0.62 & 15 & 2.8 & - \\
\hline & & P-LN J & & 0.57 & 15 & 2.1 & \\
\hline & & LS fit to LN & $6.9 \pm 0.22$ & $0.59 \pm 0.023$ & 15 & 1.9 & 0.98 \\
\hline \multirow{9}{*}{$67 \pm 6.6$} & $1 d$ & Poisson ) & $5.7^{\S}$ & - & 7 & 1.1 & \\
\hline & & LN & & 0.54 & 6 & 5.6 & - \\
\hline & & P-LN I & & 0.28 & 6 & 0.66 & \\
\hline & & Constrained, LS fit to LN & $5.7 \S \pm 0.33$ & $0.48 \pm 0.044$ & 5 & 15 & 0.88 \\
\hline & & LS fit to LN & $6.5 \pm 0.29$ & $0.52 \pm 0.033$ & 6 & 9.5 & 0.95 \\
\hline & Convolved $(0.25,1 \mathrm{~d})$ & Poisson & 5.7 & - & 14 & $9.6 \mathrm{E}+06$ & \\
\hline & & LN & & 0.62 & 13 & 8.4 & - \\
\hline & & P-LN & & 0.49 & 13 & 1.7 & \\
\hline & & LS fit to LN & $6.7 \pm 0.25$ & $0.54 \pm 0.029$ & 13 & 6.3 & 0.97 \\
\hline \multicolumn{8}{|c|}{$\begin{array}{l}\text { *Errors are based on SD of triplicate measurements of scintillation counts and cell counts. } \\
{ }^{\dagger} \text { SEs are provided by LS fit of data to LN function using SigmaPlot. } \\
{ }^{\ddagger} \text { Optimized value of } \sigma \text { is obtained via minimization of } \hat{\chi}^{2} \text { procedure. } \\
\S_{<}<n>\text { with } \S \text { is decay-corrected for } t_{2}>t_{1} ; \text { otherwise, }<n>\text { is obtained from raw data. } v \text { is degree of freedom. Values are shown up to } 2 \\
\text { gnificant digits. } \\
\text { Analysis }{ }^{210 P o} \alpha \text {-particle track distribution is based on data from Neti and Howell (7). }\end{array}$} \\
\hline
\end{tabular}

information about the overall shape of the entire activity distribution in the cell population. Accordingly, although an LN distribution with $\sigma=0.8$ fits these data extremely well, it is not appropriate to apply this distribution to this subset of data. For the same reason, the P-LN distribution cannot be used to assess the significance of the Poisson distribution in these data. This makes it difficult to tease the possible Poisson influence out of the underlying distributions in the $t=7$ and $26 \mathrm{~d}$ datasets. With this limitation in mind, it is worth revisiting the convolved data where these distributions are applicable. The 52-d data were used for the low multiplicity of convolved tracks per cell (1-9 tracks), the 26-d data were used for the middle multiplicity (5-15 tracks), and the 7-d data were used for high multiplicity (10-32 tracks). Within the overlapping regions (Fig. 1D), considerable agreement exists between the data points from independent datasets. Furthermore, an $\hat{\chi}^{2}$ analysis of the convolved data gives $7.7 \times 10^{7}, 3.6$, and 12 for the Poisson,
LN, and P-LN. The difference between the convolved data and the 3 predicted probability curves can be seen in the inset of Figure 1D at very small probabilities. Again, the data are best described by the LN distribution (Table 1). This suggests that the convolved $t=7 \mathrm{~d}$ and $26 \mathrm{~d}$ data follow the expected trend of the LN distribution, and any Poisson component in these data has a negligible impact on the overall distribution.

\section{Statistical Analysis for Cells Exposed to $3.8 \mathrm{kBq} / \mathrm{mL}$}

An approach similar to the one described earlier in this study was also applied to the 2 discrete datasets that were obtained at a labeling concentration of $3.8 \mathrm{kBq} / \mathrm{mL}$. The 2 sets of track distributions were acquired from autoradiographs that were developed at $t=0.67$ and $4 \mathrm{~d}$, as shown in Figures 2A and 2B. In this case, the $t=4 \mathrm{~d}$ dataset is the primary dataset, covers the range of 0-9 tracks of convolved data, and primarily dictates the shape of the probability 


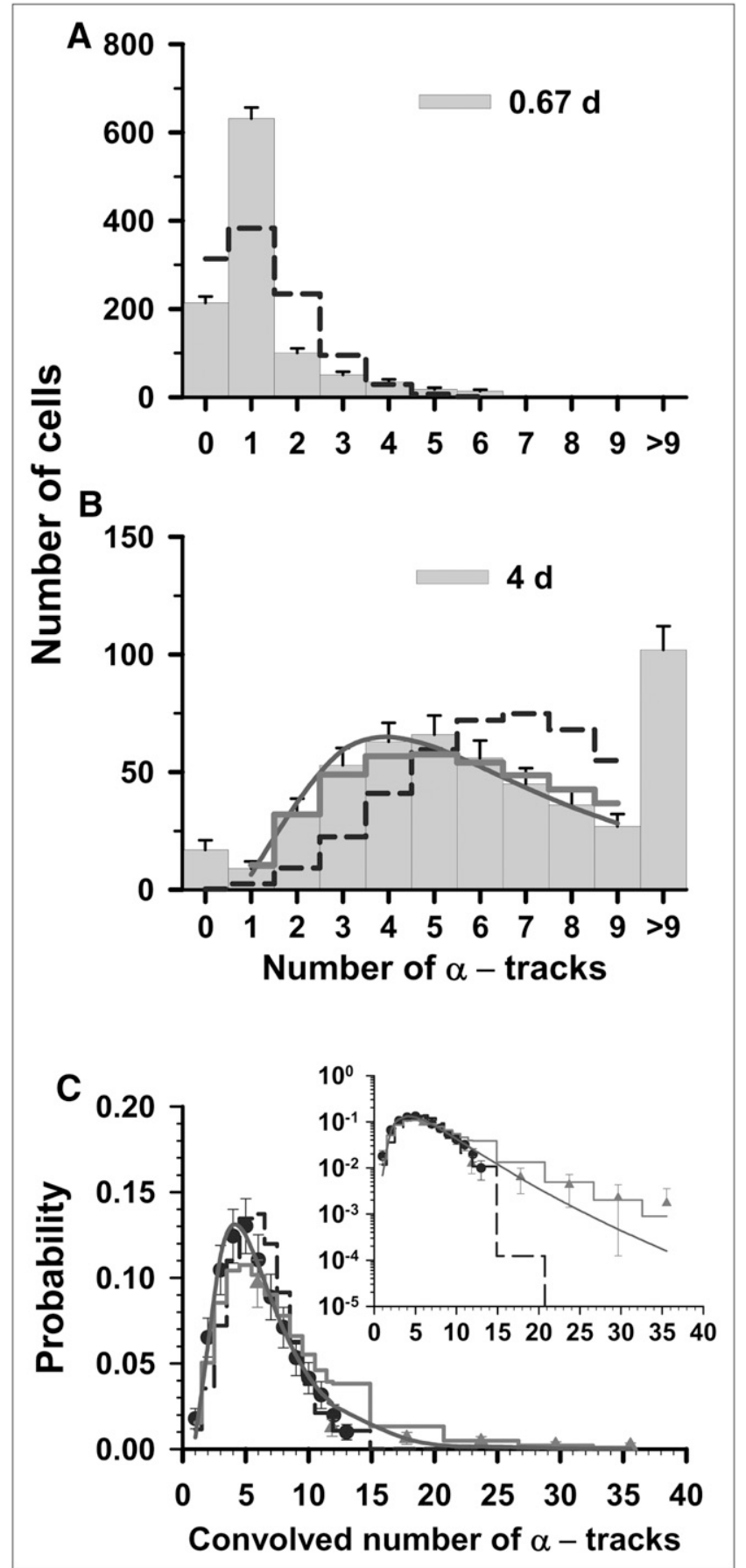

FIGURE 2. Statistical analysis of $\alpha$-particle track distribution in V79 lung fibroblasts that were labeled in culture medium containing $3.8 \mathrm{kBq}$ of ${ }^{210} \mathrm{Po}$-citrate per milliliter. (A and $\left.\mathrm{B}\right)$ Decays were allowed to accumulate for $0.67 \mathrm{~d}(\mathrm{~A})$ and $4 \mathrm{~d}(\mathrm{~B})$. (C) Data points represent normalized convolution of experimental track data obtained at $0.67 \mathrm{~d}(\boldsymbol{\Delta})$ and $26 \mathrm{~d}(\bullet)$. Error bars represent SEs. In each panel, predicted probabilities based on Poisson, P-LN, and LN functions are represented by dashed step line, thick solid step line, and solid curve, respectively. Inset plots ordinates on log scale to compare trends of these probability functions relative to experimental data at high numbers of convolved tracks. Parameters of 3 probability density functions are enumerated in Table 1. distribution. The $\langle n>$ for the $t=0.67 \mathrm{~d}$ data, where all cells have fewer than 9 tracks per cell, is 1.2 tracks per cell. The mean number of tracks per cell for the 4-d data can be obtained with Equation 1, which results in $\langle n\rangle=7.3$. The Poisson probabilities for each of the 0-9 discrete tracks per cell are calculated for $\langle n\rangle=1.2(t=0.67 \mathrm{~d})$ and $\langle n\rangle=$ $7.3(t=4 \mathrm{~d})$ (Figs. 2A and 2B). The $\hat{\chi}^{2}$ values for the Poisson distribution are 96 for the $0.67-\mathrm{d}$ and 25 for the 4-d datasets. The $\hat{\chi}^{2}$ values for all 3 distribution functions are given in Table 1. On minimization of $\hat{\chi}^{2}$, the values of $\sigma$ were 0.64 and 0.60 for the $\mathrm{LN}$ and P-LN, respectively. The lowest $\hat{\chi}^{2}$ value was obtained for the LN distribution (Table 1), thereby again suggesting that Poisson corrections are not required for the $t=4 \mathrm{~d}$ data. Furthermore, the $\sigma$ obtained for the LN distribution corresponding to the $t=4 \mathrm{~d}$ data was the same as that obtained by an LS fit to the convolved data. Agreement exists between the data points within the overlapping regions of the convolved datasets (Fig. 2C). The arithmetic mean $(<n>)$ value of 6.1 for the convolved dataset was obtained from an LS fit of the convolved data to the LN distribution. Furthermore, $\hat{\chi}^{2}$ analysis of the convolved data gives $1.2 \times$ $10^{7}, 2.8$, and 2.1 for the Poisson, LN, and P-LN. The difference between the convolved data and the 3 predicted probability curves is shown in the inset of Figure 2C. Again, the data are best described by the LS fit of the convolved data to an LN distribution function (Table $1, \hat{\chi}^{2}=1.9$ ).

\section{Statistical Analysis for Cells Exposed to $67 \mathrm{kBq} / \mathrm{mL}$}

Finally, the results are somewhat different at the highest labeling concentration of $67 \mathrm{kBq} / \mathrm{mL}$. In this case, the 2 sets of track distributions were acquired from autoradiographs that were developed at $t=0.25$ and $1 \mathrm{~d}$ (Figs. 3A and 3B). The $\langle n\rangle$ for the $t=0.25 \mathrm{~d}$ data is 1.4 tracks per cell and, using Equation $1,\langle n\rangle=5.7$ for the 1-d data. The Poisson probabilities for each of 0-9 discrete tracks per cell are calculated with Equation 2 for each $\langle n\rangle$. As shown in Figure 3A, the $t=0.25 \mathrm{~d}$ dataset does not follow a Poisson distribution $\left(\hat{\chi}^{2}=91\right)$. However, the $t=1 \mathrm{~d}$ data are better described by the Poisson distribution $\left(\hat{\chi}^{2}=1.1\right)$ than by the $\mathrm{LN}$ distribution $\left(\hat{\chi}^{2}=5.6\right)$. The P-LN distribution function provides the best fit to the data, suggesting a significant Poisson contribution $\left(\hat{\chi}^{2}=0.66\right)$. The values of $\sigma$ were 0.54 and 0.28 for the $\mathrm{LN}$ and P-LN, respectively. The $\sigma$ obtained for the LN distribution corresponding to $t=$ $1 \mathrm{~d}$ data was the same as that obtained by an LS fit for the convolved data (Table 1). Again, within the overlapping regions of the convolved data considerable agreement exists between the data points from independent datasets (Fig. $3 \mathrm{C})$. Furthermore, $\hat{\chi}^{2}$ analysis of the convolved data gives $9.6 \times 10^{6}, 8.4$, and 1.7 for the Poisson, LN, and P-LN. A comparison between the convolved data and the 3 predicted probability curves is provided in the inset of Figure 3C. The data are much better described by the LS fit of the convolved data to an LN distribution function (Table 1, $\hat{\chi}^{2}=$ 6.3) than to a Poisson distribution $\left(\hat{\chi}^{2}=9.6 \times 10^{6}\right)$, but the data are best explained by the P-LN model $\left(\hat{\chi}^{2}=1.7\right)$. 


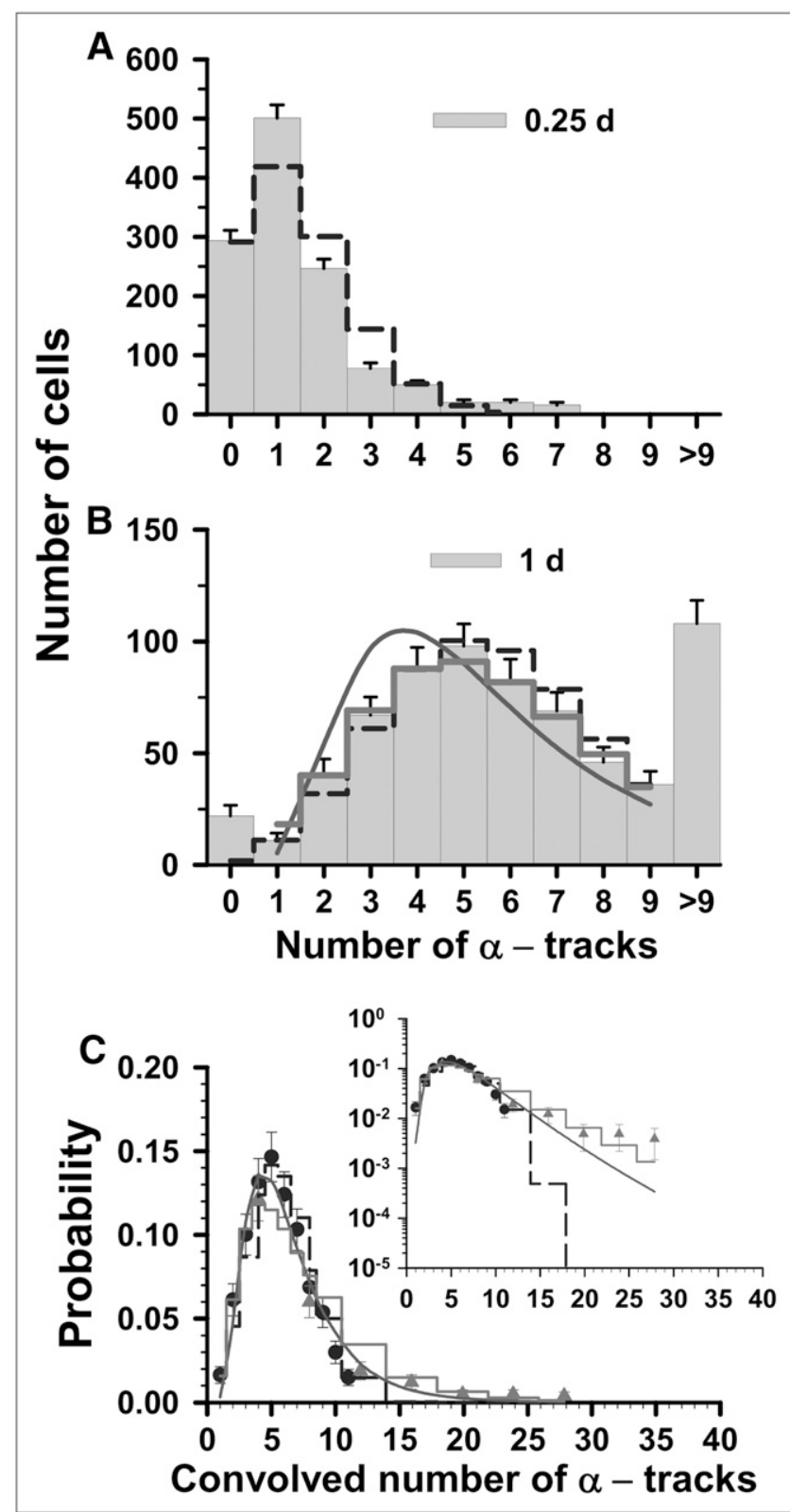

FIGURE 3. Statistical analysis of $\alpha$-particle track distribution in V79 lung fibroblasts that were labeled in culture medium containing $67 \mathrm{kBq}$ of ${ }^{210} \mathrm{Po}$-citrate per milliliter. (A and B) Decays were allowed to accumulate for $0.25 d(A)$ and $1 d(B)$. (C) Data points represent normalized convolution of experimental track data obtained at $0.25 \mathrm{~d}(\mathbf{\Delta})$ and $1 \mathrm{~d}(\bullet)$. Error bars represent SEs. In each panel, predicted probabilities based on Poisson, P-LN, and $L N$ functions are represented by dashed step line, thick solid step line, and solid curve, respectively. Inset plots ordinate on log scale to compare trends of these probability functions relative to experimental data at high numbers of convolved tracks. Parameters of 3 probability density functions are enumerated in Table 1.

\section{DISCUSSION}

As described above, a variety of approaches were used to analyze our ${ }^{210} \mathrm{Po} \alpha$-particle track distribution data. These involve statistical analyses of both the raw track data and the convolved track data using Poisson, LN, and P-LN functions. The results, summarized in Table 1, indicate that the Poisson distribution alone does not adequately describe the observed track distributions as it would if the cellular activity was distributed equally among all the cells in the population. In fact, with the exception of the data corresponding to the highest concentration of ${ }^{210} \mathrm{Po}$, a pure $\mathrm{LN}$ distribution generally provides the best description of the data. In the case of the highest concentration $(67 \mathrm{kBq} / \mathrm{mL})$, the data are best described by the P-LN distribution, which suggests that the Poisson statistics associated with radioactive decay play a significant role in the analysis of this set of autoradiographic data. Nevertheless, the underlying distribution of activity in this cell population is well described by an LN distribution.

It is interesting to more closely examine the conditions under which Poisson statistics play a significant role in interpreting our $\alpha$-particle track data. Figures 1C, 2B, and 3B show that the autoradiographic track data appear to become more Poissonian as the concentration of ${ }^{210} \mathrm{Po}-$ citrate in the culture medium is increased. This is supported by the $\hat{\chi}^{2}$ values for the Poisson, LN, and P-LN distributions. For the $1-\mathrm{d}$ dataset $(67 \mathrm{kBq} / \mathrm{mL})$, the corresponding values are 1.1, 5.6, and 0.66 (Table 1). The reason for the increased Poisson influence at higher concentrations of ${ }^{210} \mathrm{Po}$-citrate may be related to a decrease in the time interval for the observation (i.e., short-emulsion exposure time). However, the $\hat{\chi}^{2}$ values for Poisson fits to the shortinterval $0.25-\mathrm{d}(67 \mathrm{kBq} / \mathrm{mL})$ dataset and the $0.67-\mathrm{d}(3.8$ $\mathrm{kBq} / \mathrm{mL}$ ) dataset are 91 and 96 (data are not shown in Table 1). These high values indicate a poor fit, which suggests that the time interval may not be a significant factor.

It is also possible that the Poisson distribution plays a bigger role with increasing concentration of ${ }^{210} \mathrm{Po}$-citrate because of a reduction in nonuniformity in the distribution of radioactivity among the cell population. To further pursue this possibility, the value of the $\mathrm{LN} \sigma$ obtained by the various methods described above are graphically compared in Figure 4. The dashed line connects what we believe are the best values for $\sigma$, where best is defined as the lowest $\hat{\chi}^{2}$ (Table 1 ). On the basis of the linearity of the 3 data points on a logarithmic scale, the $\sigma$ is exponentially dependent on the extracellular concentration. A decreasing value of $\sigma$ implies more uniform distribution of radioactivity among the cell population. This implies that the nonuniformity of the cellular uptake of ${ }^{210} \mathrm{Po}$-citrate decreases with increasing extracellular concentration $(7,8)$. Figure 6 in an earlier study (7) shows that this will result in a more exponential survival curve rather than the saturating dose-response curves that are expected for $\mathrm{LN}$ distributions with large $\sigma$ values.

In our earlier article, we used the convolution approach to analyze the experimental $\alpha$-particle track data (7). Kvinnsland et al. expressed concern about the effect of the convolution approach on the impact of Poisson statistics on the resulting distribution (9). The overlapping regions of convolved data from independent datasets (Figs. 1D, 2C, and 


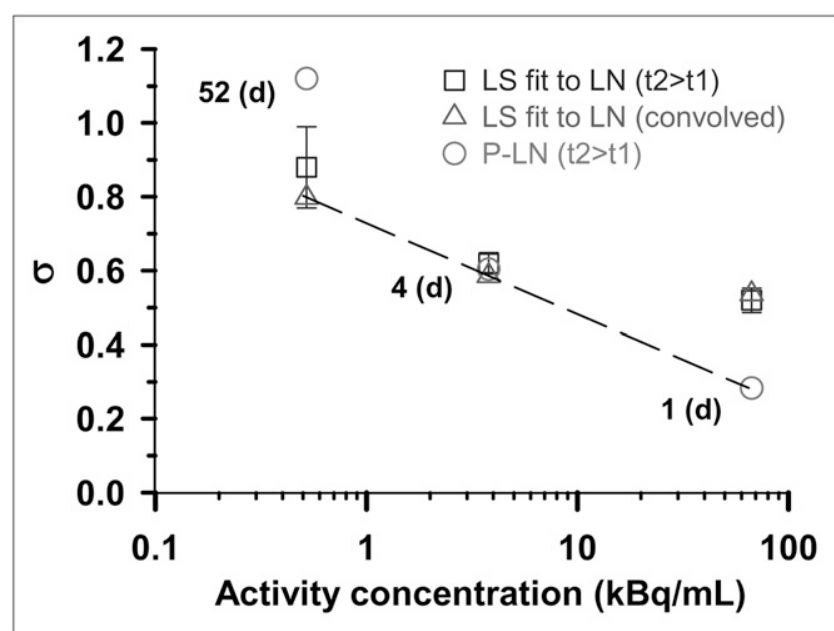

FIGURE 4. LN $\sigma$ for 3 different activity concentrations of ${ }^{210} \mathrm{Po}$-citrate. Open squares $(\square)$ represent values of $\sigma$ obtained, when $\langle n\rangle$ was constrained, by LS fits of experimental track data corresponding to longest decay accumulation times (i.e., $t_{2}>t_{1}$, Figs. 1C, 2B, and $3 B$ ) to LN distribution function. SEs of these fitted $\sigma$-values are indicated by vertical lines. Corresponding $\sigma$-values obtained for these same data using P-LN distribution function are shown as open circles $(\bigcirc)$. Open triangles $(\triangle)$ correspond to LS fits of convolved data (Figs. $1 \mathrm{D}, 2 \mathrm{C}$, and $3 \mathrm{C}$ ) to $\mathrm{LN}$ function. Finally, dashed line passes through what are considered best $\sigma$-values as defined by lowest $\hat{\chi}^{2}$.

3C) suggest that the data can be convolved. The $\sigma$ obtained by LS fit of the primary dataset (1-9 tracks, $t_{2}>t_{1}$ ) and the convolved data to an $\mathrm{LN}$ function are also similar for the cases of 0.52 and $3.8 \mathrm{kBq} / \mathrm{mL}$, which supports the convolution approach as well. However, as shown in Figure 4, the convolution approach is not satisfactory for the case of 67 $\mathrm{kBq} / \mathrm{mL}$ datasets. In this instance, as described above, the underlying LN distribution must be extracted from the measured distribution using the P-LN function. Thus, caution must be exercised when implementing the convolution approach. The $\sigma$ values obtained directly from the 52-d data and the 4-d data are very similar to those values obtained from the convolved data. Therefore, provided that one has a single autoradiograph that covers most of the track distribution (e.g., Figs. 1C or 2B), one may be able to obtain $\sigma$ without resorting to convolution.

To test the potential use of $\mathrm{LN}$ distributions to describe the cellular uptake of other radiochemicals within a population of cells, the data of Lehman and Scavia are revisited (18). Their cells were labeled with ${ }^{33} \mathrm{P}$, an emitter of $\beta$-particles with a mean energy of $76.9 \mathrm{keV}$, under unstirred and stirred conditions, and the frequency distribution of autoradiographic grains per cell was measured. Lehman and Scavia noted that the distribution of background grains per cell was adequately described by a Poisson distribution, but the experimental treatments (stirred and unstirred mixtures) were not explained by a simple Poisson distribution (18). Consequently, they used a Neyman's Type A distribution, which consists of a composite of 2 Poisson processes (one process that describes grain-counting statistics and another process that pertains to the randomness of radioactive decay (19)). They fit their data to this distribution and obtained $\hat{\chi}^{2}$ values of 0.68 and 1.7 for the stirred and unstirred conditions, respectively. To assess the capacity of the $\mathrm{LN}$ distribution to describe their data, the arithmetic mean $(\langle n\rangle)$ was calculated for each mixture, and an $\hat{\chi}^{2}$ analysis was conducted for Poisson, LN, and P-LN. Furthermore, an unconstrained LS fit of the data to an $\mathrm{LN}$ function was performed. The results of these fits show that the LN, P-LN, and LS fit to LN generally yield similar values of $\hat{\chi}^{2}$ and LN $\sigma$ (Table 2). Thus, the underlying distribution of radioactivity among the cell population is well described by an LN distribution.

The analyses above support the conclusion that the distribution of radioactivity in a cell population can often be well represented by an LN distribution. LN distributions have been shown to describe a variety of natural processes. Bulmer (20) fitted the species abundance data, which showed that truncated, grouped LN distributions provided a satisfactory fit over the logarithmic and P-LN models, omitting zero class. Other models exist that can be useful for explaining data with a priori knowledge of the underlying distribution, such as multivariate P-LN distributions (21), bivariate Poisson distributions (19), and so on. However, LN distributions are ubiquitous and have been observed across many fields $(22,23)$.

Finally, this exercise was undertaken to demonstrate the statistical significance of the $\mathrm{LN}$ distribution that was obtained for ${ }^{210} \mathrm{Po}$-citrate using an $\alpha$-particle track autoradiographic approach (7). The reliability of this approach stems from its use of emitted radiations to ascertain the distribution. Other experimental techniques, such as flow

TABLE 2

Relevant Parameters for $\beta$-Particle Tracks per Cell in Autoradiography Experiment Along with Quantities of Statistical Tests for Stirred and Unstirred Mixtures

\begin{tabular}{|c|c|c|c|c|c|}
\hline \multirow[b]{2}{*}{ Type and function } & \multirow[b]{2}{*}{$<n>^{*}$} & \multirow[b]{2}{*}{$\sigma^{\dagger}$} & \multirow[b]{2}{*}{$v$} & \multicolumn{2}{|c|}{$\begin{array}{c}\text { Statistical } \\
\text { test }\end{array}$} \\
\hline & & & & $\hat{\chi}^{2}$ & $R^{2}$ \\
\hline \multicolumn{6}{|l|}{ Stirred mixture } \\
\hline Poisson & 8.7 & - & 22 & 0.15 & - \\
\hline LN & 8.7 & 0.70 & 20 & 0.0071 & - \\
\hline P-LN & 8.7 & 0.80 & 20 & 0.0064 & - \\
\hline LS fit to LN & $12 \pm 1.4$ & $0.79 \pm 0.075$ & 20 & 0.0048 & 0.79 \\
\hline \multicolumn{6}{|l|}{ Unstirred mixture } \\
\hline Poisson & 7.8 & - & 21 & 0.99 & - \\
\hline LN & 7.8 & 0.67 & 20 & 0.0077 & - \\
\hline P-LN & 7.8 & 0.68 & 20 & 0.0046 & - \\
\hline LS fit to LN & $8.2 \pm 0.37$ & $0.57 \pm 0.033$ & 20 & 0.018 & 0.93 \\
\hline
\end{tabular}

*SEs are provided by LS fit of data to $L N$ function using SigmaPlot.

${ }^{\dagger}$ Optimized value of $\sigma$ is obtained via minimization of $\hat{\chi}^{2}$ procedure.

The $\langle n\rangle$ and $\sigma$ are obtained from raw data based on Lehman and Scavia (18) and minimization of $\hat{\chi}^{2}$ value. 
cytometry $(24,25)$, can also be used to derive the distribution. Because of the ubiquitous presence of LN distributions, many investigators studying radiobiologic responses to radiopharmaceuticals and other radiochemicals may find this distribution useful to fold into their dose-response models $(8,24,26)$. Its implementation is facilitated by several factors. First, it is an analytic function that is described by only 2 parameters $(\sigma, \mu)$. Second, the LN probability density function is provided in standard subroutine libraries (e.g., National Algorithm Group) for computational purposes.

\section{CONCLUSION}

Statistical tests show that the LN distribution function is favored as a general form to describe the distribution of cellular uptake of ${ }^{210} \mathrm{Po}$ among a population of cells exposed to the same concentration of ${ }^{210} \mathrm{Po}$-citrate. Furthermore, this distribution is likely to be applicable to a variety of radiopharmaceuticals.

\section{ACKNOWLEDGMENT}

This work was supported in part by U.S. Public Health Service grant R01CA83838.

\section{REFERENCES}

1. Leblond CP, Wilkinson GW, Belanger LF, Robichon J. Radio-autographic visualization of bone formation in the rat. J NIH Res. 1997;9:44-55.

2. Soremark R, Hunt VR. Autoradiographic studies of the distribution of polonium210 in mice after a single intravenous injection. Int J Radiat Biol. 1966;11:43-50.

3. Jönsson B-A, Strand S-E, Larsson BS. A quantitative autoradiographic study of the heterogeneous activity distribution of different indium-111-labeled radiopharmaceuticals in rat tissues. J Nucl Med. 1992;33:1825-1832.

4. Roberson PL. Quantitative autoradiography for the study of radiopharmaceutical uptake and dose heterogeneity. J Nucl Med. 1992;33:1833-1835.

5. Humm JL, Macklis RM, Bump K, Cobb LM, Chin LM. Internal dosimetry using data derived from autoradiographs. J Nucl Med. 1993;34:1811-1817.
6. Akabani G, Kennel SJ, Zalutsky MR. Microdosimetric analysis of alphaparticle-emitting targeted radiotherapeutics using histological images. $J$ Nucl Med. 2003;44:792-805.

7. Neti PVSV, Howell RW. Log normally distributed cellular uptake of radioactivity: implications for biological responses to radiopharmaceuticals. $\mathrm{J} \mathrm{Nucl}$ Med. 2006;47:1049-1058.

8. Neti PVSV, Howell RW. Biological response to nonuniform distributions of ${ }^{210}$ Po in multicellular clusters. Radiat Res. 2007;168:332-340.

9. Kvinnsland Y, Stokke T, Aurlien E. Log normal distribution of cellular uptake of radioactivity. J Nucl Med. 2007;48:327.

10. Neti PV, Howell RW. Reply: log normal distribution of cellular uptake of radioactivity. J Nucl Med. 2007;48:327a-328a.

11. Handel PH. Quantum approach to 1/f noise. Phys Rev A. 1980;22:745-757.

12. Kousik GS, Gong J, van Vliet CM, et al. Flicker noise fluctuations in $\alpha$-radioactive decay. Can J Phys. 1987;65:365-375.

13. Azhar MA, Gopala K. Search for $1 / \mathrm{f}$ fluctuations in $\alpha$ decay of ${ }^{210}$ Po. Phys Rev A. 1989;39:5311-5313.

14. Miller G. Statistical modelling of Poisson/log-normal data. Radiat Prot Dosimetry. 2007;124:155-163.

15. Aitchison J, Brown JAC. The Log-Normal Distribution. Cambridge, U.K.: Cambridge University Press; 1957.

16. Crow EL, Shimizu K. Lognormal Distributions: Theory and Applications. New York, NY: Marcel Dekker; 1988.

17. Fors O, Núñez J, Richichi A. CCD drift-scan imaging lunar occultations: a feasible approach for sub-meter class telescopes. Astron Astrophys. 2001;378: 1100-1106.

18. Lehman JT, Scavia D. Microscale nutrient patches produced by zooplankton. Proc Natl Acad Sci USA. 1982;79:5001-5005.

19. Johnson NL, Kotz S. Discrete Distributions. Boston, MA: Houghton-Mifflin; 1969.

20. Bulmer MG. On fitting the Poisson lognormal distribution to species-abundance data. Biometrics. 1974;30:101-110.

21. Aitchison J, Ho $\mathrm{CH}$. The multivariate Poisson-log normal distribution. Biometrika. 1989;76:643-653.

22. Mitzenmacher M. A brief history of generative models for power law and lognormal distributions. Internet Mathematics. 2004;1:226-251.

23. Limpert E, Stahel WA, Abbt M. Log-normal distributions across the sciences: keys and clues. Bioscience. 2001;51:341-352.

24. Kvinnsland Y, Stokke T, Aurlien E. Radioimmunotherapy with alpha-particle emitters: microdosimetry of cells with a heterogeneous antigen expression and with various diameters of cells and nuclei. Radiat Res. 2001;155:288-296.

25. Pinto M, Howell RW. Concomitant quantification of targeted drug delivery and biological response in individual cells. Biotechniques. 2007;43:64, 66-71.

26. Ballangrud AM, Yang WH, Palm S, et al. Alpha-particle emitting atomic generator ${ }^{225}$ Actinium-labeled trastuzumab (herceptin) targeting of breast cancer spheroids: efficacy versus HER2/neu expression. Clin Cancer Res. 2004;10: 4489-4497. 